\title{
Environmental Scanning Electron Microscopy in Transmission Mode
}

\author{
H. Dobberstein, ${ }^{*}$ O. A Homsy, ${ }^{*}$ D. E. Morrison, ${ }^{*}$ and B. L. Thiel** \\ *Department of Physics, Cavendish Laboratory, University of Cambridge, Cambridge, CB3 0HE, \\ UK. \\ **College of Nanoscale Science and Engineering, University at Albany - SUNY, 255 Fuller Road, \\ Albany, NY 12203.
}

The motivation for using a STEM detector in scanning electron microscopy is three-fold: first, to consider any novel transmission contrast mechanisms, which would result from the relatively strong electron-specimen interactions occurring at the low beam energies in a SEM, as compared to conventional transmission electron microscopy (TEM) and scanning transmission electron microscopy (STEM); additionally to use bright and dark field imaging in transmission mode (Figure 1), and the possibility of a higher resolution obtained with a STEM detector than in standard ESEM detectors. Secondly, to exploit the low vacuum environment for examining specimens incompatible with high vacuum, including insulators (charging), and materials containing water (biomembranes, cells), and finally, as an inexpensive alternative to standard transmission microscopy (TEM or STEM).

The STEM detector has been developed by FEI Company and a schematic of this detector is shown in Figure 2. Essentially it consists of a two-segment, solid-state backscattered electron detector over which one suspends a TEM-style thin-film specimen. Positions 2, 3, 4, 6, 7 \& 8 place the specimen entirely over one segment only, resulting in a pseudo-bright field transmission/absorption image including all but high-angle scattered electrons $\left(>56^{\circ}\right)$. The positions 1 and 5 place the specimen off-centre partially over both segments. When measuring the signal from segment B, only electrons scattered through a reasonably large angle are collected and thus results in a dark field image. The signal from segment A includes all electrons except those scattered through very large angles $\left(>56^{\circ}\right)$, resulting in an image similar to that of the single-segment positions $(2,3,4,6,7,8)$. The signal from segment $\mathrm{A}$ is not a true bright field signal, and something approximating a true bright field image can be obtained by appropriate scaling and subtraction of signal B from A.

The STEM detector can be operated in both high vacuum and low vacuum conditions. However, if the detector is used in high gas pressures, it has been found that the gas environment in the sample chamber can degrade the picture quality as illustrated in Figure 3. This lead to this study, which compares the quality of STEM micrographs after varying ESEM operating conditions, such as beam energy $(\mathrm{kV})$, beam current, working distance, gas pressure, and gas type. The obtained images were analyzed using the free software Scion Image Beta 4.02 (Scion Cooperation) and the plug-in macro SMART, which is written by D. C. Joy [1] and can be downloaded from his webpage [2]. The software package employs a Fast Fourier transform to generate a diffractogram (two dimensional power spectrum) of the image, which allows the quantitative analysis of important electron microcopy parameters, namely, the probe size, resolution, and signal to noise ratio.

In this presentation, we will show results of the analytic STEM study, and also compare them to images obtained by traditional STEM and TEM. Further, the potential of the STEM detector and its drawbacks will be briefly discussed, as there has been only little work presented in the past [3]. 


\section{References}

[1] D. C. Joy, et al., Proc. SPIE, 3998 (2000) 108.

[2] http://web.utk.edu/ srcut.

[3] D. E. Morrison, et al., Proc. SCANNING, 26 (2004) 77.
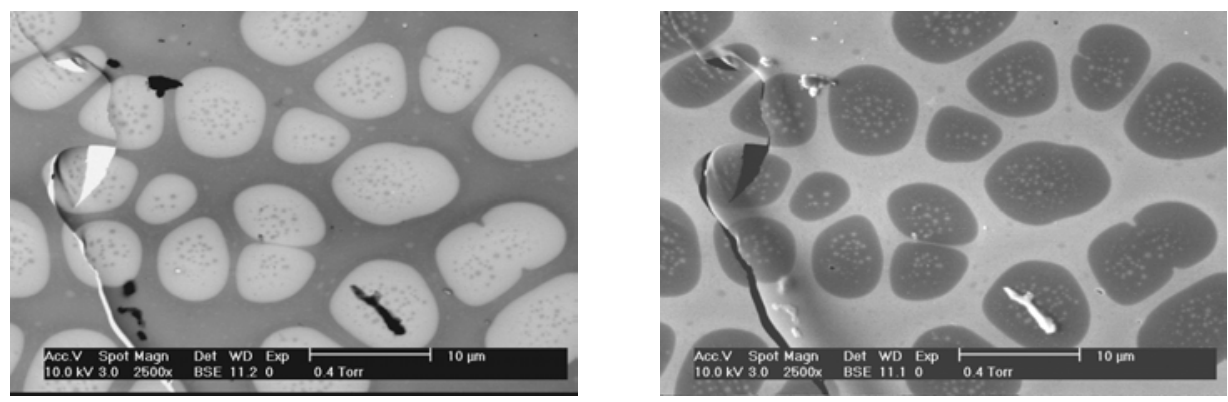

FIG. 1. Bright field (left) and dark field (right) STEM image pair from a thin film of semiconducting polymer blend. (PFB domains and the F8BT matrix. Conditions: $10 \mathrm{keV}, 0.4$ torr).

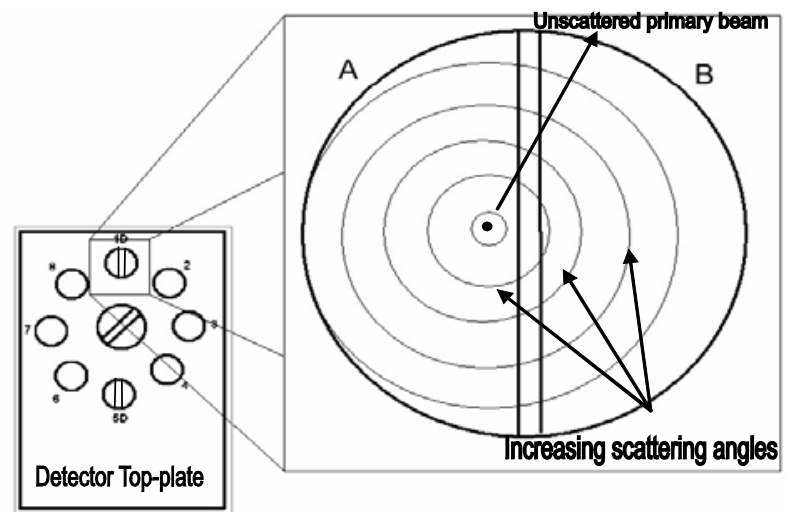

FIG. 2. Schematic of the transmission detector. The detector assembly consists of a plate with spaces for eight TEM-type specimens (left), situated of a two-segment backscattered solid-state electron detector. Two of the positions (top and bottom in this figure) were positioned to span both segments of the detector, as indicated on the right hand side. Concentric circles represent increasingly larger scattering angles.
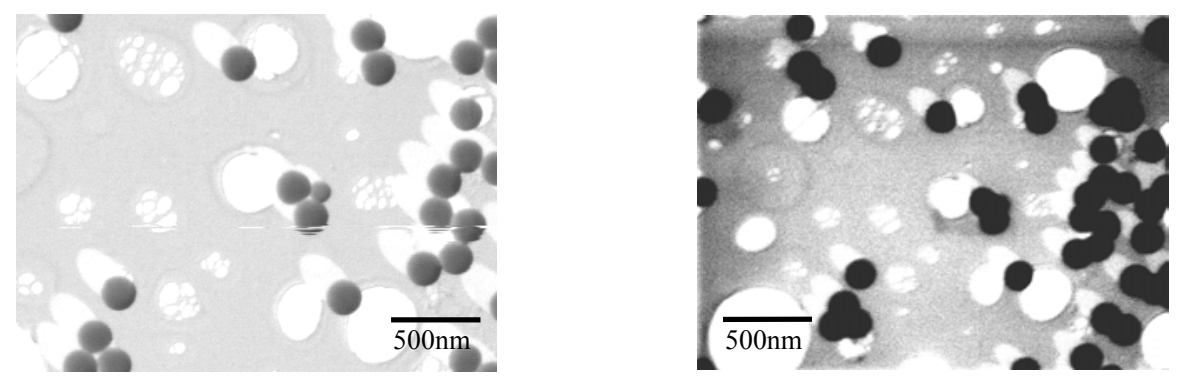

FIG. 3. Bright-field micrographs of latex balls (gold shadowed) on carbon coated TEM grits. The left image is taken at 0.0 torr $(10 \mathrm{keV}, 4 \mathrm{~mm} \mathrm{WD})$; the right image is obtained at 3 torr $(10 \mathrm{keV}, 4 \mathrm{~mm}$ WD). 